\title{
COMMISSIONING OF THE SLS DIGITAL BPM SYSTEM
}

\author{
V. Schlott, M. Dach, M. Dehler, R. Kramert, P. Pollet, T. Schilcher, PSI, Villigen, Switzerland; \\ M. Ferianis, R. DeMonte, Sincrotrone Trieste, Italy; \\ A. Kosicek, R. Ursic, Intrumentation Technologies, Slovenia.
}

\begin{abstract}
During the commissioning of the Swiss Light Source (SLS) first operational experience has been gathered with the newly developed digital beam position monitor (DBPM) system. The DBPM performance could be characterized in all available operation modes, which are: single turn, turn-by-turn, closed orbit and tune. All modes can be selected independently for each sector of the booster and storage ring through the EPICS-based SLS control system by simple re-programming of the digital processing chain. Laboratory measurements as well as measurements on the SLS accelerators will be presented, offering insights in the operational experience and the performance of the DBPM system.
\end{abstract}

In addition, a monitoring system (POMS) for the transverse mechanical positions of each BPM block in reference to the adjacent quadrupole magnets has been installed and commissioned. First results will be presented, showing a horizontal and vertical movement of the BPM blocks as a function of beam current (heat load on the vacuum chamber) in the SLS storage ring.

\section{INTRODUCTION}

The commissioning of the SLS [1] was strongly supported by the newly developed digital BPM system. Applying the concept of digital receivers [2], it is possible to operate only a single, four channel electronics in different operation modes for all accelerators of the SLS complex. Mode selection is realized by simply reprogramming the quad digital receiver (QDR) [3] through the EPICS-based control system, providing maximum flexibility to the operators. Direct data transfer to beam dynamics applications [4] allows automated measurement procedures and fast and efficient analysis of accelerator physics phenomena. Presently, the data transfer is arranged through a batch process consisting of up to 8192 $\mathrm{x}-$ and $\mathrm{y}$-position as well as intensity readings, which are synchronized to the $3 \mathrm{~Hz}$ repetition rate of the SLS injector. With the high bandwidth / medium resolution turn-by-turn mode, injection in the booster synchrotron and storage ring has been optimized and important lattice parameters like tunes, chromaticities and beta values have been measured to high precision [1]. Determination of closed orbits and beam based alignment have been performed with the low bandwidth/high resolution closed orbit mode. A self test mode has been established to monitor mal-functioning of electronics modules. The application of a pilot signal in the RF front end [3] allows calibration of the four channel electronics through individual gain setting.

\section{DBPM PERFORMANCE}

The performance of the DBPM system has been measured under stable conditions (external RF source) in the laboratory using a similar set-up as for the SLS storage ring. Resolution in different operation modes, beam current dependence and long term stability have been determined as key features for the use of the DBPM system in a light source like SLS. Each measurement has been performed with a set of pre-calibrated gain levels, which guarantee that the electronics is always operated in its linear regime. It is planned to implement an automated gain control (AGC) in the future, when all systematic effects of the electronics are completely understood.

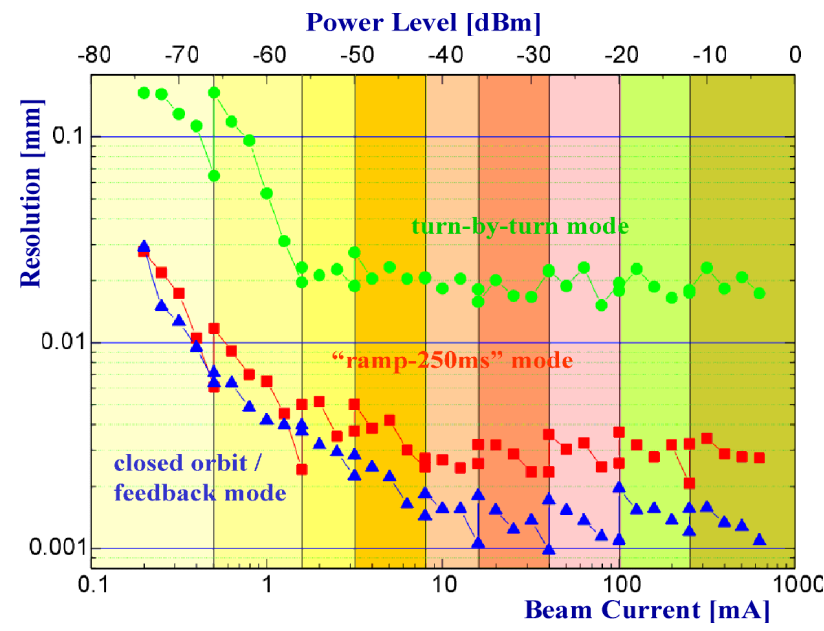

Figure 1: Measurement of DBPM resolution for different bandwidths: $500 \mathrm{kHz}$ (green dots), $15 \mathrm{kHz}$ (red squares) $2 \mathrm{kHz}$ (blue triangles). Gain levels of the system are kept constant for the marked beam current ranges.

The resolution measurements agree well with the expectations [5]. At low beam currents (high gains), the noise contribution in the RF front end dominates the resolution of the system, while at higher beam currents, the limitation is given by the ADC resolution. Still, a low bandwidth noise floor can be observed, showing up more clearly in the low bandwidth operation modes. It is suspected, that it is caused by phase noise in the RF front end, which is presently under investigation. Nevertheless, 
the minimum resolution in different operation modes of the DBPM system scales with the square root of bandwidth and is measured to be $<20 \mu \mathrm{m}$ in turn-by-turn mode $(1 \mathrm{MS} / \mathrm{s}),<3 \mu \mathrm{m}$ in the so called "ramp-250ms" mode $(30 \mathrm{kS} / \mathrm{s})$ and $<1.2 \mu \mathrm{m}$ in the closed orbit mode $(4 \mathrm{kS} / \mathrm{s})$. The sudden decreases of resolution whenever the gain levels are changed is a systematic effect and will be minimized as soon as the AGC loop is operational.

Beam current dependence has been measured in the lab for a centered beam by performing several measurement cycles over the whole dynamic range of the system with the same (constant) pre-calibrated gain settings as above. At low beam currents substantial jumps in position occur, whenever a new gain setting is loaded, which indicates, that the calibration routine still needs to be improved. At beam currents above $5 \mathrm{~mA}$ the drifts are $< \pm 5 \mu \mathrm{m}$ and within the specifications of SLS.

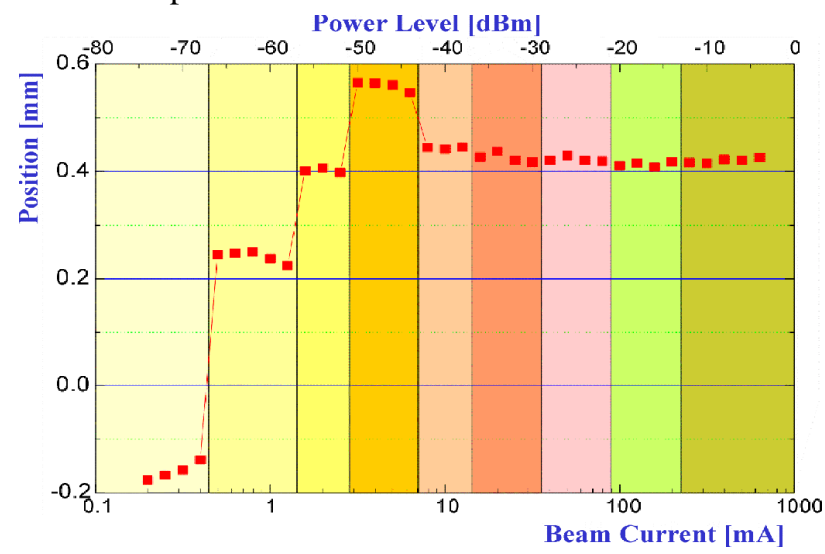

Figure 2: Beam current dependence over the whole dynamic range of the DBPM system.

Long term stability has been measured with a constant input power level of $-12 \mathrm{dBm}$ and a constant DBPM gain setting at the carrier frequency of $499.654 \mathrm{MHz}$ over a time period of 24 hours. It resulted to be within $\pm 1 \mu \mathrm{m}$.

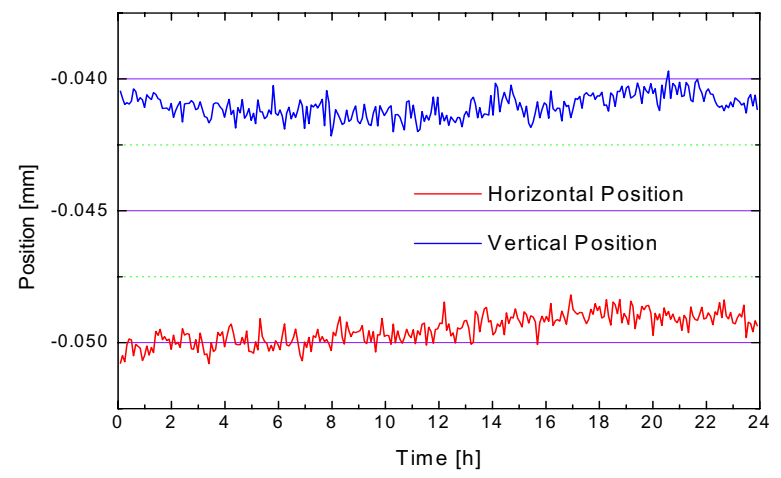

Figure 3: Long term stability of the SLS DBPM system is within $\pm 1 \mu \mathrm{m}$ over a time period of 24 hours.

\section{OPERATIONAL EXPERIENCE}

Complete integration of the DBPM system in the EPICS-based SLS control system allowed from the beginning of the commissioning the use of all available operation modes. In the pre-injector LINAC and the transfer lines, beam positions were measured in a so called pulsed mode, delivering one position reading every injection cycle. Injection in the booster synchrotron as well as in the storage ring has been optimized using the turn-by-turn mode of the system. Tunes are constantly determined by applying a FFT to the 8192 position readings coming from a dedicated electronics. The direct data transfer from the DBPM system to the beam dynamics applications allows a fast and efficient measurement of important lattice parameters like chromaticities and beta values, which in return provide excellent understanding of the storage ring optics. Consequently, the machine model of the SLS storage ring is extremely close to reality, providing the ability to correct closed orbits to only a few micron in both planes with reference to any established "golden" orbit.

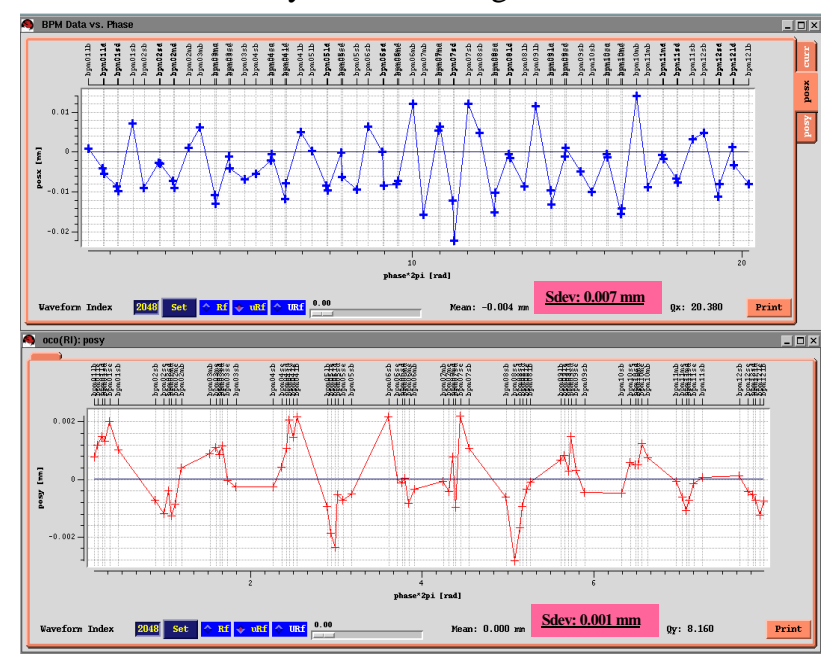

Figure 4: Horizontal (top) and vertical (bottom) orbits in SLS storage ring. The correction was performed to $7 \mu \mathrm{m}$ rms and $1 \mu \mathrm{m}$ rms in reference to a "golden" orbit, which leads to minimum heat load on the vacuum system.

The power spectrum of beam motions in the SLS storage ring has been extracted from DBPM data, taken in the high resolution / low bandwidth, closed orbit mode without further averaging. Two sets of measurements were performed with the booster synchrotron on and off to study its influence on the beam in the storage ring (red and green curves in figure 5). In both planes only minor beam motions can be detected at $3 \mathrm{~Hz}$, corresponding to the SLS injection cycle. In the frequency range between $15 \mathrm{~Hz}$ and $30 \mathrm{~Hz}$ beam motions are measured, which coincide well with the eigenfrequency spectrum of the SLS storage ring girders, excited by ground motions. Finally, $50 \mathrm{~Hz}$ and odd harmonics (not displayed in figure 5) are also visible in the power spectrum. The measurements, show that the resolution of the DBPM system in the storage ring is sufficient to detect beam motions up to $2 \mathrm{kHz}$, which indicates, that the SLS global closed orbit feedback [6] should be able to correct beam movements up to $100 \mathrm{~Hz}$ as specified. 


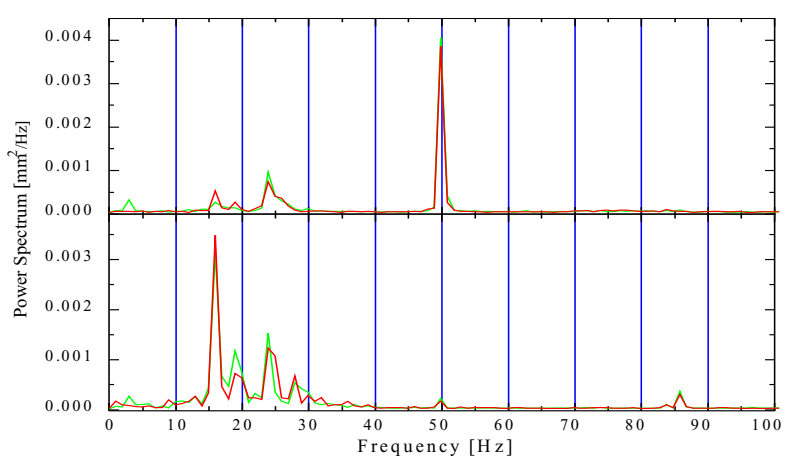

Figure 5: Power spectrum of beam motions in the SLS storage ring with booster on (green) and off (red).

\section{BPM BLOCK POSITIONS}

Finite element analyses (FEA) show, that thermal loads or gradients caused by changing beam currents in the storage ring, lead to strong deformations of the vacuum chamber, resulting in transverse BPM block motions in the order of $2 \mu \mathrm{m} /{ }^{\circ} \mathrm{K}$. This effect has been observed during storage ring commissioning with a so called mechanical position monitoring system (POMS) [7], using two linear encoders of the Renishaw RGH24Z50A00A type with $0.5 \mu \mathrm{m}$ resolution per BPM station. Figure 6 shows the movement of some of the BPM stations in reference to the adjacent quadrupoles as a function of beam current for a typical situation during storage ring commissioning. Even for well corrected orbits, the (horizontal) position drifts are in the order of some tens of microns (up to $50 \mu \mathrm{m}$ with $150 \mathrm{~mA}$ current change), which agrees well with the FEA-simulations.

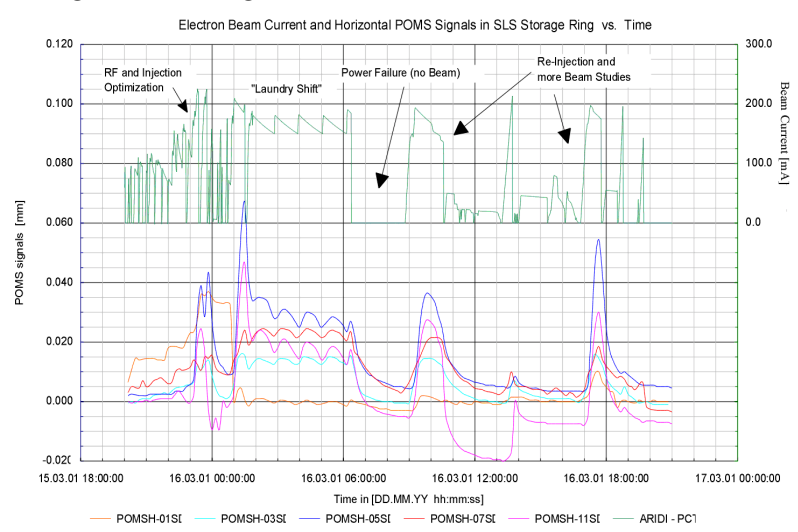

Figure 6: Horizontal BPM block movements as a function of beam current during SLS storage ring commissioning.

Since BPM readings are always relative measurements between the sensors and the beam itself, it can be expected that any BPM block movement shows up in the BPM data in opposite direction. Figure 7 shows this effect for a night shift, where beam was freshly injected into a "cold" machine and kept constant over a period of 8 hours. After collecting more experience with the POMS system, it is finally planned to use these data for the determination of the "real", absolute beam positions. This should allow to keep any "golden" orbit over time and under changing heat load conditions, improving the reproducibility and stability of the SLS as a user facility.

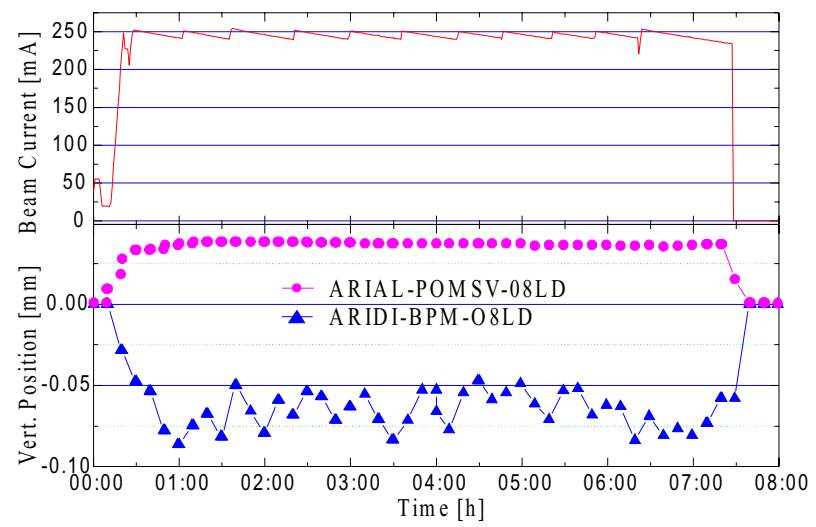

Figure 7: Top: electron beam current in storage ring during night shift. Bottom: vertical BPM block movements, measured with POMS and compared to DBPM readings.

\section{CONCLUSIONS AND OUTLOOK}

The DBPM system has reached and even exceeded the design specifications in all available operation modes. Its complete integration into the EPICS-based SLS control system allows online re-programming of the hardware including features like operation mode selection and electronics calibration. Direct transfer of position data to beam dynamics applications supports a large number of measurement procedures, providing good understanding of lattice parameters. Mechanical movements of the BPM blocks have been observed with the POMS system. The order of magnitude of some tens of microns agrees well with FEA-simulations. Corresponding depositions to the opposite direction in the DBPM readings support these observations. DBPM features like an AGC loop and real time data transfer for the SLS closed orbit feedback will be implemented in the next few months in order to support SLS user operation, starting in August 2001.

\section{REFERENCES}

[1] A.Streun, et al. "Commissioning of the Swiss Light Source", contribution to this conference.

[2] R. Ursic et. al, "Digital Receivers offer new Solutions for Beam Instrumentation", proc. PAC'99, New York, 1999, p. 2253.

[3] V. Schlott, et al. "Digital BPM System for the Swiss Light Source - First Operational Results", AIP Conf. Proc. 546, BIW'00, Cambridge, May 2000, p. 572.

[4] M. Böge, et al. "Commissioning of the SLS using CORBA based Beam Dynamics Applications", contribution to this conference.

[5] M. Dehler et. al, "BPM System for the Swiss Light Source", proc. PAC'99, New York, 1999, p. 2087.

[6] M. Böge, et al. "Fast Closed Orbit Control in the SLS Storage Ring" PAC'99, New York, June 1999.

[7] V. Schlott, "Monitoring Mechanical Drifts at SLS using Linear Encoders" PSI Annual Report 1999, SLS Annex, p. 19. 